\title{
Evaluaciones Formativas en el Aula: Análisis Discursivo de la Actividad de Retroalimentación en la Práctica Supervisada de Psicólogos Educativos en Formación
}

\author{
Formative Assesment in the Classroom: Discursive Analysis of \\ the Feedback Activity in Supervised Practice of Undergraduate \\ Educational Psychologists
}

\author{
Jorge Alberto Moreno Ruiz *1 \\ Antonia Candela Martín ${ }^{1}$ \\ Patricia Bañuelos Lagunes ${ }^{2}$
}

${ }^{1}$ Centro de Investigación y Estudios Avanzados del Instituto Politécnico Nacional, México

${ }^{2}$ Universidad Nacional Autónoma de México, México

\begin{abstract}
Este es un estudio discursivo sobre las evaluaciones formativas ocurridas en una actividad de retroalimentación como parte de la Práctica Supervisada de estudiantes de psicología educativa en una universidad pública de México (UNAM). Aunque se ha reconocido el valor que tiene la formación en la práctica de los psicólogos, hay pocos estudios que muestren cómo ocurren las prácticas de evaluación formativa en el aula. Debido a ello, nuestro objetivo es analizar los procesos de trabajo interaccional involucrados en una actividad de retroalimentación, cuando los estudiantes se convierten en evaluadores de la competencia o habilidades psico-educativas, en modalidades de la evaluación formativa como (a) la coevaluación, (b) la evaluación entre pares y (c) la autoevaluación. Para ello, analizaremos diversos episodios interaccionales que ocurren en el aula, después que ha ocurrido una primera sesión de orientación psico-educativa en una cámara de Gesell, y los participantes (maestra, pasante y estudiantes) se reúnen y evalúan la competencia profesional de una estudiante que fungió como psicóloga a cargo del caso. Para orientar el análisis, se recurre a un enfoque analítico discursivo, informado por la etnometodología y el análisis de la conversación, que permite examinar, momento a momento, las evaluaciones formativas sobre el trabajo de la psicóloga y los distintos posicionamientos que se hacen públicos alrededor de su desempeño. Los resultados muestran que en esta práctica supervisada se construye colectivamente una evaluación formativa en la que tanto la estudiante psicóloga encargada del caso como el pasante y el resto de las estudiantes que observaron y participaron en la evaluación tienen oportunidad de mejorar su desempeño profesional a través de la interacción discursiva que se desarrolla en el proceso. Esto permite reconocer la importancia de este tipo de análisis para estudiar la evaluación de las prácticas supervisadas y su valor formativo en situaciones reales.
\end{abstract}

Palabras clave: Evaluación formativa; Práctica educativa; Supervisión; Retroalimentación; Análisis del discurso; Psicólogos educativos.

This is a discursive study of the formative assessments as feedback activities that take place as part of the Supervised Practice of undergraduate educational psychologists at a Mexican public university (UNAM). The formative practice of undergraduate psychologists has been recognized as very important, however there are few studies that show how are they evaluated as a formative practice at the classroom. Analyze the processes of interactional work involved in a feedback activity, when students become evaluators of competence or psycho-educational skills, in modalities of formative assessment such as (a) co-evaluation, (b) peer evaluation and (c) self-evaluation, is this work purpose. After the first psycho-educative session at the Gesel camera several classroom interactional extracts were analysed, where the participants (the teacher, an intern and undergraduates) evaluate the professional competence of an undergraduate student that work as the psychologist of a particular case. A discursive approach informed by the ethnometodology and the conversation analysis perspective orients the study in order to exam, turn by turn, the formative assessments of the undergraduate psychologists work and the public positions about her performance. The analysis results show the collective construction of the formative assessments of the supervised practice realised by

*Contacto: psiquea24@yahoo.com.mx

issn: 1989-0397

www.rinace.net/riee/

https://revistas.uam.es/riee
Recibido: $\quad 16$ de enero de 2019

$1^{\text {a }}$ Evaluación: 04 de febrero de 2019

$2^{\text {a }}$ Evaluación: 31 de marzo de 2019

Aceptado: $\quad 01$ de abril de 2019 


\begin{abstract}
a psychologist student. This psychologist together with a passant are in charge of the case and the rest of the students observe and participate in the assessment of the supervised practice. Through these collective discursive processes, all the participants have the opportunity to improve their professional performance. This results show the important contribution of this kind of analysis in order to describe and recognize the formative contribution of the supervised practices evaluated in real settings.
\end{abstract}

Keywords: Formative assessment; Educational practice; Supervision; Feedback; Discourse analysis; Undergraduate educational psychologists.

\title{
1. Introducción
}

La investigación sobre la formación de psicólogos educativos en contextos en que los estudiantes tienen acceso a la práctica profesional supervisada es muy limitada. En México sólo unos pocos estudios se han centrado en esta parte de la formación de psicólogos en las universidades y son escasos los que muestran la forma en que ocurren las evaluaciones en el aula. Esto quiere decir que hay poco conocimiento de las prácticas concretas que constituyen las actividades (como la retroalimentación) en las que pueda mostrarse parte del trabajo realizado para formar a los futuros psicólogos educativos.

Este estudio busca mostrar la manera en que el acceso a la práctica profesional supervisada juega un papel en las prácticas de evaluación formativa que llevaron a cabo un grupo de estudiantes, su maestra y un pasante, en una clínica universitaria donde se brinda atención y se forma a estudiantes en el área de psicología educativa en la FES-Z. Así, se pretende contribuir a mostrar de qué manera ocurre la formación de los psicólogos educativos de forma detallada, y específicamente, la manera en que llevan a cabo algunas modalidades de la evaluación formativa, como son la coevaluación, la evaluación entre pares y la autoevaluación, con respecto al trabajo de orientación que brindan a la comunidad.

\section{Fundamentación teórica}

\subsection{La investigación de la práctica y la evaluación formativa en la formación de psicólogos educativos en México}

Desde hace casi tres décadas, el psicólogo César Coll (1989) había mencionado la importancia de asegurar el contacto directo de los estudiantes con la práctica profesional del psicólogo de la educación. Al respecto, hay poca investigación sobre la manera en que los estudiantes tienen contacto directo con la práctica profesional cuando se encuentran estudiando.

Guzmán (2005) por ejemplo, destaca que "hay poca información para saber exactamente cómo se forma tanto al psicólogo general como al educativo" (p. 7). En el caso específico de la psicología educativa, Díaz, Hernández, Rigo, Saad y Delgado (2006) reiteran la falta de estudios empíricos y actuales sobre la formación de psicólogos educativos como una problemática actual:

No disponemos de un corpus de conocimiento empírico y actual, situado en nuestra realidad respecto a los procesos de aprendizaje profesional y académico mediante los cuales una persona se convierte en un psicólogo competente en los escenarios educativos. (p. 21)

Estos autores, consideran "que las competencias sólo pueden ser adquiridas en la práctica, realizando las tareas, responsabilidades y roles requeridos", "en un proceso de "aprender haciendo" en la situación actual del trabajo" (p. 16). También, reconocen que, "diversas 
instituciones abocadas a la enseñanza de la Psicología se han destacado por sus modelos de formación profesional basados en la práctica en escenarios naturales" (p. 15); tal es el caso de las escuelas de la UNAM: Facultad de Psicología, FES Iztacala y FES Zaragoza. En estas escuelas, "los estudiantes, asesorados y supervisados por su profesor, identifican la situación que aqueja a la población y proporcionan alternativas de solución a problemas semejantes a los que encontrarán en su futuro desempeño profesional" (Buenrostro, 2016, p. 2).

Al respecto, Díaz (2006), señala que, desde una perspectiva de la enseñanza situada, la evaluación auténtica que se destaca en las situaciones que se propician en estos escenarios, es la que evalúa los aprendizajes contextualizados, que captura "la riqueza de lo que los alumnos han logrado comprender, solucionar o intervenir” (p. 127).

Un ejemplo de lo anterior se refiere a "las prácticas auténticas de intervención" que se realizan en clínicas de salud mental, en las cuales, los alumnos desarrollan o perfeccionan "las competencias propias de dicha comunidad de práctica profesional” (Díaz, 2006, p. 127). Siguiendo con el ejemplo de lo que hacen los estudiantes en una clínica, cuando ellos tienen que hacerse cargo de una entrevista inicial, pueden identificarse las prácticas de evaluación auténticas cuando se analiza colectivamente en el grupo, lo que hizo la estudiantepsicóloga durante la entrevista. Es aquí, que las evaluaciones formativas pueden ocurrir.

Algunas modalidades de éstas, que Díaz (2006), encuentra distintivas son: la evaluación mutua (o evaluaciones entre pares), la coevaluación (que estipula una confrontación de las evaluaciones realizadas por el docente y el estudiante) y la autoevaluación fundamentada por el alumno.

En estas modalidades, según Díaz (2006), lo importante es la identificación del vínculo de coherencia entre lo conceptual y lo procedural, o seguir el proceso de apropiación de determinados saberes o formas de actuación de la comunidad de práctica profesional.

Sobre esta base, Hernández (2007) identifica que, en la actualidad, en el campo de la psicología educativa se inicia una fase donde apenas se comienzan a estudiar los procesos educativos. Lo que este autor sugiere es recurrir a técnicas y estrategias metodológicas de corte cualitativo que permitan abrir

Nuevas posibilidades para desarrollar un trabajo de investigación que permita reconocer la
complejidad de las realidades educativas (como procesos institucionales, culturales y sociales),
que considere las perspectivas de los participantes estudiados..., así como la especificidad de
los contextos, sin pretender alterarlos arbitrariamente por fines "científicos". (p. 29)

Sobre este tipo de investigación cualitativa, algunos autores (por ejemplo, Rodríguez y Seda, 2013) han comenzado a estudiar las prácticas educativas enfocándose en lo que significa haber tenido acceso a la práctica profesional de psicólogos educativos durante su formación, recuperados de reportes obtenidos a través de entrevistas. Puede decirse que ellos han buscado las experiencias formativas en la voz de los propios psicólogos en formación.

Si bien se reconoce que esta es una vía para el estudio de la práctica de los psicólogos en formación, queda mucho por hacer para lograr tener el "corpus de conocimiento empírico y actual, situado en nuestra realidad” (p. 21) al que aludían Díaz y colaboradores (2006). Esto es similar en los estudios sobre las prácticas de evaluación en el aula en México, 
cuyos datos se conocen sólo a través de cuestionarios y entrevistas a docentes y estudiantes por separado (Martínez y Mercado, 2015, p. 19)

De acuerdo con Ravela, Picaroni y Loureiro (2017), uno de los obstáculos para crear el "corpus' de conocimiento profesional" (p. 41), que no sólo atañe a campo disciplinar de la psicología educativa, es que "la docencia es una actividad predominantemente solitaria" (p. 40). Para estos autores, "es necesario moverse de la visión de la enseñanza como actividad solitaria... hacia una visión de la enseñanza como una actividad profesional abierta a la observación, el estudio y el mejoramiento colectivos" (p. 43).

Más aún, el estudio de las evaluaciones formativas que implica considerar también al estudiante, necesita recuperar su actuación en una comunidad de práctica profesional, como también lo señalaban Díaz y colaboradores (2006). La alternativa, por tanto, son investigaciones empíricas centradas en procesos interactivos dentro del aprendizaje situado, como la propuesta por Candela (2018), quien estudia grupos de pares como parte de una comunidad académica en la educación superior.

En la siguiente sección, se presenta la orientación teórico-metodológica que orientó este estudio de la práctica educativa, también cualitativo, pero centrado en las evaluaciones formativas a través un análisis discursivo informado etnometodológicamente.

\section{Metodología}

\subsection{Enfoque teórico-metodológico}

En este apartado, describimos la manera en que el análisis discursivo informado etnometodológicamente investiga cualitativamente la práctica educativa. La etnometodología y el análisis de la conversación han logrado generar un cuerpo sustancial de investigación que usa el video para estudiar el logro práctico e interaccional de las actividades en los lugares de trabajo que sitúan a la "práctica" en el corazón de la agenda. A través de este foco, se indaga la manera en la cual los participantes producen, dan sentido a, y coordinan, las actividades de manera conjunta. Estos compromisos analíticos, asociados con la gama sustancial de ideas, observaciones y hallazgos sostienen al creciente corpus de estudios basados en videograbaciones de las prácticas del trabajo profesional (Heath, Hindmarsh y Luff, 2010).

$\mathrm{El}$ análisis discursivo informada etnometodológicamente toma ventaja de la visibilidad de la acción práctica; es decir, atiende su carácter observable-reportable y describe los campos públicos en los cuales trabajan los participantes en escenarios educativos. Así, la atención está puesta en lo que los psicólogos educativos expresan y cómo actúan, para mostrar el conocimiento técnico con el que trabajan. Macbeth (2003) también se refiere a este enfoque como uno que permite análisis naturalistas de los escenarios educativos, cuya concepción de las competencias y habilidades en escenarios educativos es caracterizada por el estudio de las habilidades y competencias como visibles, públicas, y concretas, en contraste a considerarlas como ocultas, privadas, y accesibles solamente a través de análisis formales y conjeturas teóricas. Esta investigación se encuentra más interesada en mostrar cómo se logra el trabajo a través del cual se forma a los psicólogos educativos ordinariamente en la práctica, que en cómo debería hacerse idealmente como lo sugieren las teorías y modelos prescriptivos. 
Desde esta perspectiva, las declaraciones sobre cómo diseñar las prácticas de enseñanza y aprendizaje pueden tomarse en consideración, pero es prioritario investigar y recuperar la racionalidad endógena y el carácter naturalmente explicable de la interacción. Así, más que investigar para diseñar "buenas prácticas", se tiene en consideración, que las prácticas no están determinadas por las teorías, modelos o concomfanciaeptos. Las teorías y modelos son idealizaciones generales, mientras que las prácticas son llevadas a cabo in situ alterando, en el contexto, tales ideales.

Esta forma de estudiar las prácticas de evaluación formativa hace frente a las múltiples formas que pueden tomar en la práctica. Como indica Zorrilla (2017), "no hay una forma única, ni mejor para evaluar en el aula", debido a que la evaluación se construye "a partir del contexto particular del docente y de sus estudiantes", y de este modo, "los mecanismos de evaluación no pueden ser fijos ni homogéneos porque cada grupo es diferente" (p. 16). A pesar de esto, a juicio de Ravela, Picaroni y Loureiro (2017), "mientras se invierten grandes cantidades de tiempo y dinero en la realización de evaluaciones a gran escala, son casi inexistentes los recursos y la atención destinada a mejorar las evaluaciones que ocurren cotidianamente dentro del aula" (p. 31).

De acuerdo a lo anterior, proponemos que antes, o al mismo tiempo que se intentan mejorar las evaluaciones que ocurren en el aula, sería importante estudiarlas directamente, como sugiere Candela (2018): de forma interactiva.

Con este contexto, partimos de que uno de los aspectos a considerar para estudiar la manera en que ocurren las evaluaciones formativas en el aula, es atender a la descripción verbal de los participantes, cuando hacen referencia a personas. Debido a ello, es importante prestar atención a la manera en que se realizan las formulaciones. Por ejemplo, según Lerner, Bolden, Hepburn y Mandelbaum (2012), puede indagarse la manera en que un hablante hace referencia o caracteriza una acción cuando la adscribe a una persona. Este tipo de indagación es lo que desde el análisis de la conversación se denomina el estudio de la referencia a personas.

De acuerdo a Schegloff (1996), "la referencia a personas en la conversación implica, como asunto de relevancia interaccional primaria, consideraciones del 'diseño del receptor” (p. 458). La importancia de esto, es que resulta crucial analizar la manera en que al hacer la referencia a una persona (presente o no) en la interacción, los participantes pueden orientarse de diversas formas a quién(es) es/son su(s) interlocutor(es) y qué sabe(n) sobre el referente (si la referencia es conocida o desconocida para él/ellos).

La importancia de esta línea de investigación para este estudio es que, según Clift (2006), al estudiar el tema de quién produce una evaluación primero y quién después - y así, quién está llegando a un acuerdo o discrepando con quién - los hablantes están valorando también referentes.

Para Potter (2012), en esta línea de trabajo, el énfasis analítico se encuentra en la manera en que la conducta se torna describible y los hechos se tornan factuales tanto en el habla como en el texto. Estos temas, pueden considerarse como dimensiones epistémicas de la conversación y son, el acceso epistémico (quién conoce qué), la primacía epistémica (quién tiene derecho de saber qué y quién sabe más sobre qué) y la responsabilidad epistémica (quién es responsable de saber qué). A través de su indagación, se ha reconocido que las asimetrías de conocimiento están imbuidas de una variedad de prácticas diferentes. Por 
ejemplo, a través de ellas, es posible examinar la manera en que las personas muestran tener acceso a ciertos estados de asuntos, pero también cómo se está en lo cierto respecto de lo que se sabe, la autoridad relativa y los diferentes derechos y responsabilidades con respecto a este conocimiento (Stivers, Mondada y Steensig, 2011).

Esta investigación recurre también a este campo, para mostrar la manera en que los participantes se involucran y toman posicionamientos en distintas instancias de evaluaciones formativas, es decir, cuando hacen algo más que hacer referencia: evaluar aquello a lo que se refieren.

Dada la falta de investigación en el estudio empírico y detallado de las prácticas de evaluación formativa cuando los estudiantes se involucran en ella, este estudio, entonces, se propone analizar algunos ejemplos en los que ocurren las evaluaciones formativas en el aula, justo después de haber brindado orientación psico-educativa a una familia mediante la entrevista inicial. Se pretende analizar los procesos de trabajo interaccional involucrados en una actividad de retroalimentación, cuando los estudiantes se convierten en evaluadores de la competencia o habilidades psico-educativas, en modalidades de la evaluación formativa como (a) la coevaluación, (b) la evaluación entre pares y (c) la autoevaluación.

\subsection{Trabajo de campo y construcción del procedimiento analítico}

Esta investigación se realizó en siete fases en las que puede describirse el proceso metodológico (Potter, 2012):

\section{Fase uno: Obtener acceso y consentimiento}

Para poder videograbar las actividades en el aula se contactó a una profesora del área de psicología educativa de la FES-Z, UNAM. Con la maestra, se negoció tanto el acceso a sus clases de Práctica Supervisada en una clínica multidisciplinaria, así como el consentimiento informado para poder grabar las actividades con sus estudiantes en el aula. En total, los participantes de este estudio fueron la misma maestra, un pasante y diez estudiantes de sexto semestre.

\section{Fase dos: Instrumentos de recolección de información}

Una vez que se tuvo acceso a las clases, se videograbaron dos sesiones de cinco horas cada una, durante 4 meses que duró el trabajo de campo. En este artículo, se presenta lo que ocurrió en un día de trabajo en el aula.

\section{Fase tres: Transcripción y tratamiento de los datos}

Se seleccionaron aquellos episodios interaccionales que mostraran el trabajo colectivo de las evaluaciones formativas (coevaluación, evaluación entre pares y autoevaluación) alrededor del trabajo de una psicóloga-estudiante que brindó orientación psico-educativa. Se transcribieron los diferentes intercambios discursivos y se les incorporó, cuando se consideró necesario, códigos de transcripción como los siguientes: “(())" se usan para hacer comentarios sobre las características del contexto o el habla, el signo de interrogación "?" marca una entonación interrogativa, el signo "=" marca un cambio de turno inmediato al final de la emisión del hablante actual, y, los dos puntos “:::” marcan la prolongación del sonido inmediatamente anterior. 


\section{Fase cuatro: Desarrollo del foco analítico}

Una vez que se han seleccionado los episodios interaccionales, se intentó explicar el desarrollo de la práctica educativa, y en el proceso, el foco analítico fue continuamente refinado. En este caso, se pretende analizar las evaluaciones formativas que crearon los participantes en su interacción.

Fase cinco: Desarrollo de las categorías de análisis

En la revisión de las transcripciones se van elaborando las categorías de análisis al reconocer que cuando los estudiantes se convierten en evaluadores de la competencia o habilidades psico-educativas, se pueden identificar tres modalidades de la evaluación formativa como (a) la coevaluación, (b) la evaluación entre pares y (c) la autoevaluación. Estas serán las categorías del análisis de los datos.

\section{Fase seis: El procedimiento analítico}

En términos procedimentales, primero identificamos las prácticas referenciales que regularmente tomaron la forma de descripciones de acciones que realizaba algún participante (aunque nos centramos mayormente en las referencias al trabajo de la psicóloga, como lo hicieron los participantes). Posteriormente, examinamos el trabajo interaccional que las referencias-acciones específicas estaban haciendo. Tomando en cuenta las participaciones por turnos, recurrimos a las dimensiones epistémicas de la interacción sobre algún estado de conocimiento, como son: el acceso epistémico (quién conoce qué), la primacía epistémica (quién tiene un derecho de saber qué y quién sabe más sobre qué) y la responsabilidad epistémica (quién es responsable de saber qué) para mostrar el posicionamiento de los participantes ante aquello que evaluaban.

\section{Fase siete: Desarrollo y validación del análisis}

Finalmente, el análisis se desarrolla tras varios intentos de especificar lo que está ocurriendo en la interacción. El interés principal es, si los participantes están constituyendo con sus propias acciones los fenómenos que son de interés para el investigador (por ejemplo, la ocurrencia de la coevaluación, la evaluación entre pares y la autoevaluación) y cuál es el tema que están tratando, como lo fue la competencia profesional de la psicóloga-estudiante. No obstante, la principal validación es la evaluación del lector, es decir, presentar los materiales analíticos en una forma que les permita, en el grado que le sea posible, hacer sus propios juicios. En este caso, una forma de validar el análisis fue invitar a los participantes (estudiantes) para que nos indicaran sí podrían ver su trabajo representado en nuestra descripción e interpretación analítica (incluyendo a uno de los autores de este artículo, que fue también la maestra).

\subsection{Los participantes y el contexto}

Para dar inicio a los resultados del análisis, es conveniente mostrar brevemente la distribución de los participantes en la cámara de Gesell cuando se brindó orientación psico-educativa a una familia. La Figura 1 muestra la vista superior de las dos salas de la cámara de Gesell divididas por el espejo unidireccional. Una es la sala de orientación (derecha) y la otra la sala de observación (izquierda). 


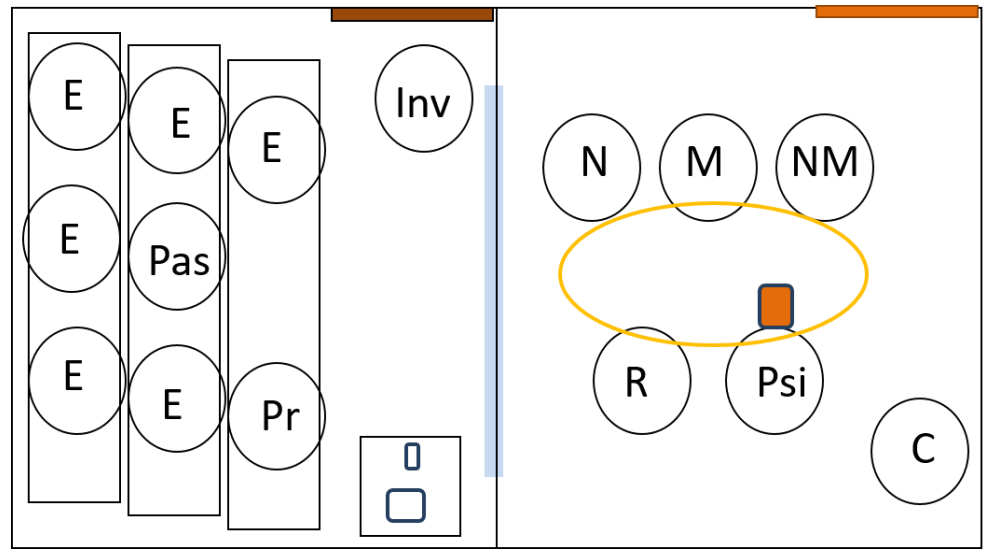

Figura 1. Distribución de los participantes en las dos salas de la cámara de Gesell Fuente: Elaboración propia.

Cabe comentar que, en la sala de orientación, como su nombre indica, ocurre la orientación psico-educativa con la familia (N: niña, M: mamá, NM: novio de mamá; véase la elipsis), y están a cargo de llevarla a cabo el camarógrafo $(\mathrm{C})$, la relatora $(\mathrm{R})$ y la psicóloga (Psi) que conforman un equipo de estudiantes. Por otra parte, en la sala de observación, la profesora (Pr), el pasante (Pas) y el resto de estudiantes (E) se encuentran escuchando y observando lo que ocurre en la sala contigua.

Después de que ha ocurrido la primera sesión de orientación en la cámara de Gesell, la estudiante que fungió como psicóloga junto con su equipo a cargo del caso, y el resto de los participantes (maestra, pasante y estudiantes), se reunieron en el aula y se involucraron en una actividad que llamaron retroalimentación y en la que discutieron sobre el trabajo profesional de la primera. Así, las estudiantes llegaron con conocimiento de primera mano sobre lo ocurrido, bien por haber brindado el servicio, o bien por haber observado y escuchado la manera en que se brindó el servicio.

Con esto en cuenta, a continuación, describiremos la manera en que los participantes comenzaron a involucrarse en evaluaciones formativas como parte de la actividad de retroalimentación.

\section{Resultados}

\subsection{La coevaluación: la evaluación del pasante sobre el trabajo de la psicóloga}

Ya en el aula, el pasante comienza a brindar retroalimentación al equipo a cargo del caso. Es importante señalar que esta era una actividad habitual del pasante que, tenía como consigna evaluar el trabajo de la psicóloga-estudiante con la ayuda de una hoja de observación que contenía los elementos que se tenían que cubrir en la entrevista inicial. El siguiente Fragmento 1 muestra lo que ocurre una vez que se comienza a grabar.

El Fragmento 1 comienza cuando el pasante indica al equipo que se cumplió con "todos los elementos de primer" "sesión" (línea 1). Esto muestra que el pasante cuenta con información que le permite saber cuáles son "todos los elementos", y mostrar que dispone de un conocimiento que le permite valorar si estuvieron presentes o no. Al final de su intervención, sugiere "que se podría buscar algo" "distinto", en cuanto a lo que parece ser 
uno de los elementos que ha valorado, sobre el "uso del lenguaje" (línea 4) y pone como ejemplo la "duda" de "si la palabra susceptible era la mejor" (línea 5).

Cuadro 1. Fragmento 1: Una retroalimentación informal: Observaciones del pasante

\begin{tabular}{lll}
\hline 1 & Pa: & $\begin{array}{l}\text { Se cumplió así: todos los, todos los elementos de primer, de primer sesión, eh, es } \\
2\end{array}$ \\
& & que realmente todas las observaciones que hice fue de, en términos de, de estuvo \\
& presente, presente, presente, o sea, todo así. Lo único que, que creo yo que se \\
4 & & podría buscar algo, algo distinto, en cuanto al uso del lenguaje, por ejemplo, me \\
5 & & quedó duda si la palabra susceptible era la mejor \\
6 & E2: & Cuándo? \\
7 & E3: & Aja, cuándo? \\
8 & Pa: & cuando le dijiste a la señora si le parecía que esto era susceptible para= \\
9 & E2: & =Ah, es que era pedagoga \\
10 & Pa: & ah sí, \\
11 & E3: & Sí, es pedagoga \\
12 & Pa: & olvídalo, olvídalo eso entonces \\
13 & E3: & Si verdad, muy elevado el nivel \\
\hline
\end{tabular}

Fuente: Elaboración propia.

Esta primera intervención, es respondida por dos de las estudiantes (E2 antes relatora, y E3 antes psicóloga, líneas 6 y 7, respectivamente), solicitando mayor especificidad sobre el momento (cuándo?) en que el pasante pudo hacer su observación. El pasante aclara el momento ("cuando le dijiste...") dirigiéndose a la estudiante (E3) que fungió como psicóloga, pero es interrumpido por E2 con una muestra de reconocimiento (“Ah", en la línea 9) y una justificación con base a la atribución de una categoría profesional a la señora (es decir, la madre): es que era pedagoga. El pasante reconoce a su vez esta respuesta e interviene nuevamente con la implicación de que no tenga en cuenta su comentario inicial: olvídalo entonces (línea 12). E3, sin embargo, hace explícita su afiliación con una posible razón de la sugerencia del pasante: si verdad, muy elevado el nivel.

Siguiendo a Raymond y Heritage (2006), como en otras secuencias de evaluación, ya que el pasante fue el primero en dar muestras de ofrecer una evaluación, aunque sea con duda (si el uso de una palabra utilizada por la psicóloga era la mejor), ello traería consigo una afirmación implícita de quién tiene mayor conocimiento sobre las acciones de la psicóloga. Sin embargo, como primer evaluador muestra cierta ambigüedad con respecto a su mayor conocimiento, pues es una evaluación sobre la que también tiene una duda. De hecho, luego de que se le pide mayor especificidad y se ofrece una razón sobre el uso de la palabra ("es que era pedagoga”), el pasante modifica su postura al degradar sus afirmaciones indicando que no las tomen en cuenta. En consecuencia, las estudiantes modifican su postura inicial de escuchar la evaluación y aclaran algo que el pasante no sabía ("era pedagoga"). Lo anterior indica que, si bien el pasante y las estudiantes tuvieron acceso a la situación referente, no contaban con la misma información.

A pesar de que miembros del equipo desafían los derechos epistémicos superiores del pasante sobre el uso del lenguaje de la psicóloga, no puede considerarse así de un modo absoluto, pues ella misma termina afiliándose con la posible razón de la duda del pasante: si verdad, muy elevado el nivel (en línea 13). Esto muestra que para los participantes supone que la persona así acreditada, cuenta con cierto conocimiento especializado, y en este sentido, puede tener el nivel para "compartir" el significado de la palabra con ellos. Por implicación, muestra que puede haber algunos términos técnicos que sería mejor no emplear con personas que no comparten un cierto nivel (al parecer, educativo). 
En términos generales, la coevaluación que ocurre en este intercambio discursivo se logra cuando el pasante intenta asumir una posición epistémica superior, similar al rol del docente. Las acciones del pasante (que no es docente ni un estudiante más) siguen la estrategia de la profesora que lo ha instruido para brindar retroalimentación apoyándose en una herramienta profesional como la hoja de observación mediante la cual puede hacer observaciones sobre el desempeño profesional de las psicólogas-estudiantes cuando han llevado a cabo la entrevista inicial. Quienes reciben retroalimentación también participan cuestionando y añadiendo información relevante para la comprensión de sus acciones.

$\mathrm{El}$ intercambio discursivo particular que siguieron los participantes representa una forma en que las distintas posiciones se reorganizan y confrontan bidireccionalmente a través de la evaluación formativa de la coevaluación.

\subsection{De la coevaluación a la evaluación entre pares: La observación-comentario sobre las preguntas múltiples}

En el siguiente Fragmento 2, el pasante termina de dar retroalimentación y cede la palabra a la profesora (Pr) quien propone una nueva trayectoria interaccional que involucra a cualquiera que quiera participar.

Cuadro 2. Fragmento 2: Cesión de derechos de la retroalimentación: El comentario sobre preguntas múltiples

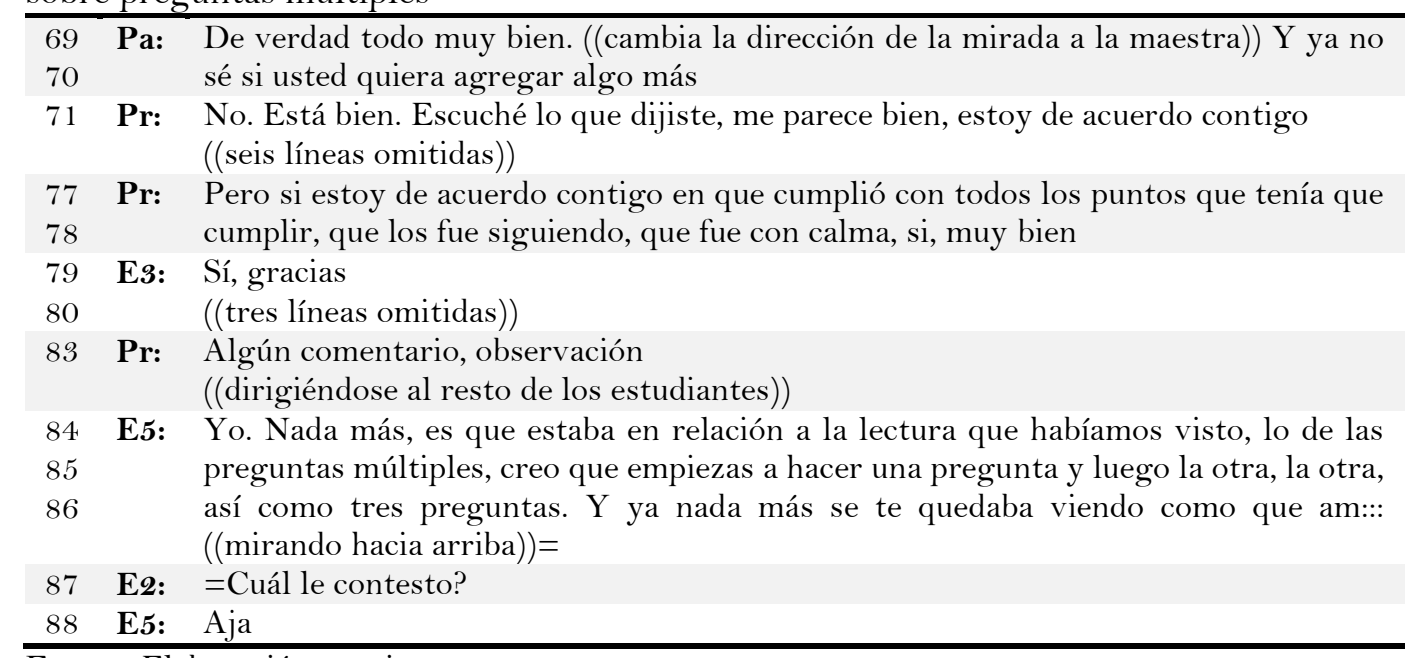

Fuente: Elaboración propia.

El Fragmento 2 inicia cuando el pasante está terminando de hacer un elogio a los miembros del equipo y continuación, se dirige a la maestra diciendo "Y ya no sé si usted quiera agregar algo más" (líneas 69-70).

Con ésta última intervención del pasante, muestra un reconocimiento del derecho de habla que en esta reunión puede tener la maestra. Cuando la maestra toma la palabra, realiza varias muestras de afiliación con lo que el pasante dijo: (1) Está bien, (2) Escuché lo que dijiste, (3) Me parece bien, y (4) Estoy de acuerdo contigo (en la línea 71). Y más adelante, reitera y hace explícitos los términos en los que concuerda con las observaciones del pasante con respecto al trabajo de la psicóloga, en tres rubros: (1) cumplió todos los puntos que tenía que cumplir, (2) los fue siguiendo, y (3) fue con calma (en las líneas 77-78). 
Con estas acciones, la maestra no solo muestra que ha monitoreado las observaciones del pasante en su evaluación del trabajo de la psicóloga, también valora el propio trabajo del pasante y de la psicóloga al hacer explícitos los términos del acuerdo. Según los analistas de la conversación Heritage y Raymond (2005), al hacer explícitos los términos del acuerdo, la maestra está haciendo también equivalentes las observaciones del pasante con las suyas; y con dichos términos, también está valorando el trabajo de la psicóloga (quien cumplió con todos puntos que tenía que cumplir y los fue siguiendo con calma) a quien termina dirigiéndose con "Sí, muy bien" indicando con ello que ha realizado adecuadamente su función como psicóloga en la entrevista inicial. Esto es reconocido por la psicóloga afirmativamente y mostrando gratitud (línea 79).

De manera similar a la sección anterior, aquí la docente evalúa las observaciones que el pasante hizo a la psicóloga, y también, evalúa el desempeño profesional de la psicóloga en la entrevista inicial. Sin embargo, en esta ocasión, ante sus evaluaciones positivas hacia ambos, ni el pasante ni la psicóloga-estudiante las desafían, en su lugar, ambos las reciben sin ningún problema y dejan intacto el estatus epistémico desde el cual, la maestra evalúa su trabajo.

Después de estas evaluaciones, la maestra abre para el resto de los estudiantes la posibilidad de que todos puedan participar con algún comentario u observación (línea 83) sobre el trabajo de la psicóloga. A partir de esta invitación por parte de la maestra, una estudiante (E5) toma la palabra y enmarca su participación como algo que "estaba en relación" a una "lectura" que habían "visto": lo de las preguntas múltiples (líneas 84-85). A continuación se dirige a la estudiante (que fungió como psicóloga) describiendo un momento en la sesión de orientación en el que hizo "como tres preguntas" (línea 86), una después de la otra, "y ya nada más" se le quedaba viendo", posiblemente la madre, "como que am::: (mirando hacia arriba). Sin que E5 hubiera terminado de actuar gestualmente, una de las integrantes del equipo, E2, menciona “¿Cuál le contesto?” (línea 87).

Con estas acciones, E5 establece primero lo que hace la psicóloga (“preguntas múltiples”), después la reacción de su interlocutora (posiblemente la madre mirando hacia arriba mientras pronuncia “am:::"), y finalmente, E2 propone "Cuál le contesto?" con lo cual muestra su interpretación de las acciones previas que la misma E5 confirma. La interpretación relevante en términos de evaluación, es que se interpreta que las preguntas múltiples que hizo la psicóloga a la madre, tuvieron el efecto en la madre de no saber cuál de ellas contestar en la entrevista inicial.

Así, E5 y E2 han emitido una valoración conjunta sobre las acciones de su par, la psicóloga. Además, E5 ha mostrado una conexión con una "lectura" que usó como fuente autorizada de conocimiento y que era familiar para todos (pues ya la "habían visto" o estudiado previamente). De esta forma, las participantes brindan una muestra de conexión entre lo que han leído ("en relación a la lectura" en la línea 84) y lo que han visto-escuchado desde la sala de observación, como una instancia de evaluación entre pares sobre lo que hizo la psicóloga en la sala de orientación. Con estas acciones, se destaca que no sólo la psicóloga puede beneficiarse de las valoraciones sobre su trabajo, también sus compañeras que emiten las valoraciones, muestran su proceso formativo al lograr interpretar las acciones de la psicóloga a la luz de la teoría contenida en una lectura, lo cual muestra el vínculo entre la teoría y la práctica que se espera que hagan las estudiantes (Díaz, 2006). 


\subsection{La autoevaluación: la coexistencia de recursos de la práctica profesional}

Para terminar, en el siguiente fragmento 3, se presenta un episodio interaccional ocurrido más tarde, en la parte final de la actividad de retroalimentación.

Cuadro 3. Fragmento 3: La valoración de las evaluaciones

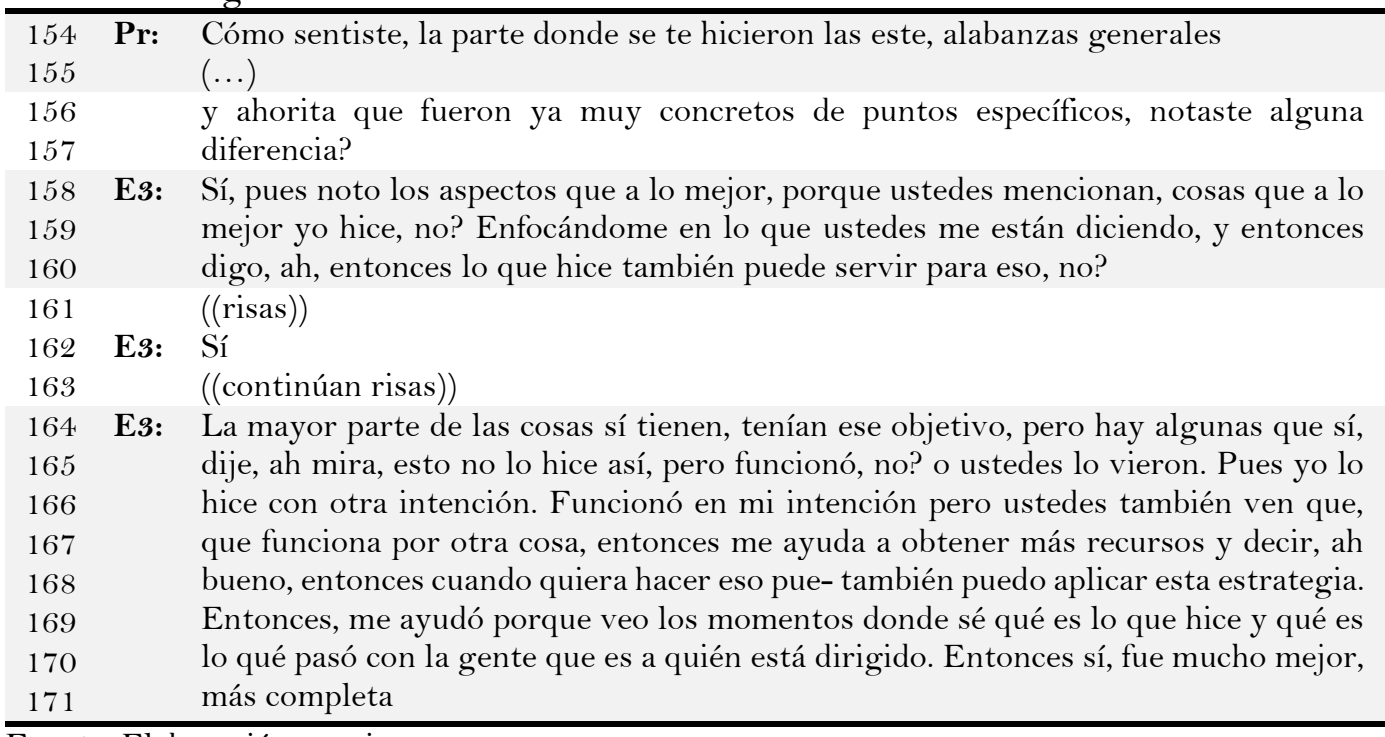

Fuente: Elaboración propia.

El fragmento 3 inicia con una pregunta que hace la maestra (líneas 154-157) en la que distingue dos actividades, y solicita que la psicóloga comente ¿cómo se sintió? Con su pregunta, la maestra confiere autoridad epistémica a la estudiante, es decir, es algo que sólo la estudiante puede responder, pues solo ella sabe cómo se sintió.

Nótese también que la pregunta proyecta una respuesta que trate sobre las evaluaciones (como las alabanzas) hechas por el resto de los participantes (maestra, pasante, compañeros) sobre el trabajo de la psicóloga en la sala de orientación, señalando que las segundas trataron "puntos específicos".

Ante la pregunta, E3, que fungió como psicóloga, ofrece dos versiones de una respuesta similar. En su primera respuesta, E3 designa lo que le han mencionado como "cosas que a lo mejor yo hice, ¿no?” (líneas 158-159). Esta primera parte de la respuesta es importante porque disminuye la factualidad de las declaraciones sobre sus acciones (a lo mejor). Sin embargo, reiterando que ha prestado atención a lo que le "están diciendo" (línea 159), ella puede decirse, "ah, entonces lo que hice también puede servir para eso, ¿no?” (línea 160).

Nótese que aquí, E3 distingue lo que le dicen de lo que se dice a sí misma, mostrando que puede haber dos formas distintas de otorgarle sentido a sus acciones, y esto se ve reforzado por la incorporación de una exclamación ante lo que le están diciendo como algo novedoso para ella: ah. No obstante, ambas partes de su respuesta incorporan al final una partícula de cortesía (no?) que puede indicar que sus declaraciones pueden requerir corroboración como respuesta. Sin embargo, a continuación, se producen risas por parte de algunos estudiantes (línea 161).

Aunque no es muy clara la razón de la producción de las risas, sí es posible apreciar que ellas fueron un incentivo para elaborar una segunda versión de su respuesta inicial. Siguiendo a Glenn (2003), al hablar en lugar de reír, E3 muestra que, no invitó la 
producción de la risa y, que no se afilió con ella (pues E3 no se ríe). Al continuar las risas por parte de sus compañeros en la línea 163, finalmente, E3 las interrumpe con su segunda respuesta, en la que reitera y expande lo que dijo, primeramente.

En su segunda respuesta, E3 expande sus declaraciones con respecto a las evaluaciones, tanto propias como ajenas, al incorporar otros términos para elaborarlas. En primer lugar, reconoce coincidencias en "la mayor parte de las cosas", pues si “tenían ese objetivo" (línea 164), pero agrega que en "algunas" "no lo hice asî" (línea 165). Así, tanto muestra que pueden interpretarse sus acciones de la misma manera (con los mismos objetivos) pero también que es posible interpretar sus acciones de un modo distinto. Esto construye a sus interlocutores y a ella misma como generadores de significados distintos atribuidos a las acciones que la psicóloga llevó a cabo en el trabajo de orientación. Ello muestra que no minimiza las contribuciones de sus compañeros. Sin desacreditar ninguna de ellas, favorece su co-existencia.

La racionalidad de la construcción permite a la hablante proyectarse a sí misma como "hacer-con-intención", y ello puede escucharse también como una auto-atribución de agencia y responsabilidad en las decisiones que tomó. En lugar de reemplazar o anular lo que la psicóloga se dice a sí misma sobre sus intenciones, lo que le dicen, expande la gama de opciones que tiene, incorporando las suyas propias, y las que otros proponen: "me ayuda a obtener más recursos" (línea 167), "cuando quiera hacer eso... también puedo aplicar esta estrategia” (línea 168).

Finalmente, ella termina señalando que la segunda actividad fue "más completa", porque le permitió ver que lo que hizo tuvo implicaciones en "lo que pasó con la gente, que es a la que está dirigido" su trabajo, reconociendo de este modo, que sus intervenciones estuvieron dirigidas a la familia y tienen un impacto en ella, y que, según la psicóloga, funcionaron. Con sus respuestas, la psicóloga muestra una auto-evaluación argumentada tanto sobre el objetivo curricular de aprender-haciendo una entrevista inicial, así como otro más profesional, como lo es tener más recursos disponibles cuando que tenga que aplicar estrategias ante problemáticas semejantes a las que enfrentará cuando actúe sin supervisión.

En términos generales, en ambas partes de su respuesta, la estudiante expresa sobre las valoraciones lo que Heritage y Raymond (2005) denominan una "orientación simétrica de primacía epistémica”. Esto quiere decir que tanto los que hacen las evaluaciones como quien las recibe tienen la misma autoridad y derechos de otorgar un significado particular a las acciones. Esto también muestra que, con estas acciones, la estudiante psicóloga se encuentra valorando las valoraciones de los otros sobre su trabajo, pero ella misma también lo está valorando. Su posicionamiento ante su conocimiento y el de los otros, muestra que el conocimiento no necesita ser el mismo, sino que al propio conocimiento puede sumarse el conocimiento de los otros, co-existiendo como recursos disponibles para la práctica profesional futura.

\section{Discusión}

A diferencia de los estudios que investigan las prácticas educativas a través de datos obtenidos de entrevistas o encuestas aplicadas a estudiantes o maestros por separado (Martínez y Mercado, 2015), el estudio más cercano al nuestro que analiza los elementos 
interactivos en una institución de educación superior como la UNAM, es el de Candela (2018), cuyo análisis interactivo sobre el trabajo entre pares de estudiantes de física, pone en primer plano las prácticas llevadas a cabo in situ, como sugirió Díaz (2006).

En nuestro caso, estudiar nociones como "competencia" o "evaluación" desde un punto de vista interaccional, son de sumo interés. Por ejemplo, a partir de los datos presentados, si se pone el foco en las evaluaciones en general, es posible ver la manera en que el pasante contó con una herramienta que le permitió observar-valorar lo que hacen las estudiantes cuando fungen como psicólogas en la actividad de orientación. Las estudiantes, sin embargo, pueden resistir las posibles evaluaciones negativas (lo que les pudo hacer falta), apelando a conocimiento que otros (como el pasante) no tienen. Las estudiantes también valoran el trabajo de sus compañeras y en el caso de quien fungió como psicóloga, el suyo propio. La maestra puede valorar el trabajo que hace el pasante sobre sus evaluaciones hacia la psicóloga-estudiante, evaluar formativamente el trabajo de la psicóloga y permitir que otras estudiantes hagan evaluaciones, incluyendo a la misma psicóloga. Estos ejemplos destacan los procesos interactivos de las evaluaciones formativas en las que se involucran los estudiantes, derivadas de enfrentar las problemáticas reales que aquejan a la familia que atienden en la entrevista inicial, similares a las que se enfrentarán en el futuro (Buenrostro, 2016; Díaz, 2006).

Consideramos que, si bien estas prácticas evaluativas tienen como referente las interacciones particulares analizadas, en otros casos también pueden ocurrir si se analiza en detalle la manera en la que los estudiantes se forman en la práctica. Esto no quiere decir que busquemos diseñar "buenas prácticas" que pueden servir de guía, ya que consideramos que ellas funcionan principalmente como prescripciones que frecuentemente no se pueden llevar a cabo en las condiciones concretas de las prácticas educativas. Debido a que nos alejamos de proponer formas de actuación idealizadas, este tipo de investigación se enfoca en estudiar las formas de actuación cotidianas, y en este sentido, también puede contribuir a generar ideas para analizar la manera en que ocurre la formación de psicólogos en otras universidades, en donde la evaluación formativa toma otras formas y tiene otras implicaciones para sus participantes (Zorrilla, 2017).

\section{Conclusiones}

Con base en los resultados del análisis, podemos decir que la evaluación auténtica, como ocurrió en la clínica donde se forma a estudiantes a través de la práctica, fue analizada a través de los ejemplos de evaluación formativa cuyas modalidades fueron la coevaluación, la evaluación entre pares y la autoevaluación.

En términos de la competencia profesional, los participantes tematizaron, principalmente, el desempeño de la estudiante cuando llevó a cabo la entrevista inicial. Los ejemplos analizados ilustraron que la coevaluación, la evaluación entre pares y la autoevaluación ocurrieron como parte de procesos interactivos en los que se abordó de diferentes maneras este tema principal, sin que la maestra fuera la única evaluadora, sino que ella misma promovió las valoraciones del resto de los participantes. En este sentido, todos los participantes tuvieron la oportunidad de atestiguar, acompañar y valorar el trabajo realizado. 
En el caso de la coevaluación, se mostró un ejemplo en el que los participantes destacaron como su tema central el uso del lenguaje de la psicóloga (como el uso de la palabra susceptible) que estuvo sujeto a discusión, y otro, en el que las evaluaciones de la maestra al pasante y a la psicóloga-estudiante no recibieron ningún desafío. Hasta este punto, las evaluaciones realizadas estuvieron encaminadas a destacar los aspectos positivos del desempeño que observaron los evaluadores.

En el siguiente ejemplo, que trató sobre la posibilidad de mejorar una habilidad relacionada con la manera de hacer preguntas, la evaluación entre estudiantes destacó que evitar hacer múltiples preguntas a su interlocutora, puede ser de utilidad para ésta última con respecto a que tendría mayores posibilidades de saber qué contestar. En este caso, el desempeño profesional se mostró como algo que se puede mejorar.

Finalmente, en la autoevaluación, el desempeño profesional no se puso en duda. En su lugar, se destacó que las acciones de la psicóloga podrían tener más de una interpretación en cuanto a su intención u objetivo. La psicóloga enfatizó que tanto las evaluaciones de otros hacia su trabajo, como su propia autoevaluación, podrían convertirse en recursos de actuación profesional futura.

También puede apreciarse que la psicóloga que se hizo cargo del caso, no fue la única que tuvo la oportunidad de mejorar su competencia. Por ejemplo, tanto su compañera de equipo (relatora) como una de sus comentaristas, llegaron a un acuerdo sobre la importancia de no hacer "preguntas múltiples" tras tomar en consideración las reacciones de la madre a quien se hicieron las preguntas y no supo cuál contestar. También pudo apreciarse, que quienes brindaron retroalimentación, como esta misma comentarista y el pasante, demostraron formas de competencia a través de las prácticas referenciales producidas en la interacción: mostraron que podían hacer conexiones conceptuales, entre las acciones de la psicóloga que ambos observaron-escucharon y los criterios de observación en la hoja del pasante y la lectura a la que aludió la estudiante y de la que todos tenían conocimiento. Esto último, es parte de su formación integral que busca vincular la teoría con la práctica (Díaz, 2006).

Para terminar, las evaluaciones formativas in situ, son un tema poco estudiado, y sabemos poco de atender las diferentes maneras de construir discursivamente a la competencia en escenarios reales, cuando se forma a los psicólogos u otros profesionistas. Sin embargo, esperamos haber contribuido, con algunos ejemplos, a la generación de un corpus de conocimiento empírico, como sugieren Diaz y colaboradores (2006), y que amplíe nuestra comprensión de cómo es que ocurren en la práctica las evaluaciones formativas de psicólogos educativos, con las singularidades y complejidades que atraviesan nuestras realidades educativas (Hernández, 2007).

\section{Agradecimientos}

Este trabajo fue realizado con el apoyo de una Beca de Conacyt.

\section{Referencias}

Buenrostro, A. (2016). Programa de apoyo al aprendizaje escolar: Docencia, servicio e investigación. Revista Electrónica de Psicología de la FES-Zaragoza-UNAM, 6(11), 1-14. 
Candela, A. (2018). Peer organized study groups: Succesful learning interactions in Mexican undergraduate physics. Learning, Culture and Social Interaction, 19, 11-21.

Clift, R. (2006). Indexing stance: Reported speech as an interactional evidential. Journal of Socioliinguistics, 10(5), 569-595.

Coll, C. (1989). Psicología académica y psicología profesional en el campo de la educación. Anuario de Psicología, 41, 49-73.

Díaz, F. (2006). Enseñanza situada: Vinculo entre la escuela y la vida. Ciudad de México: McGraw-Hill Interamericana.

Díaz, F., Hernández, G. Rigo, M., Saad, E. y Delgado, G. (2006). Retos actuales en la formación y práctica profesional del psicólogo educativo. Revista de la Educación Superior, XXXV(1), 1124.

Glenn, P. (2003). Laughter in interaction. Cambridge: Cambridge University Press.

Guzmán, J. (2005). La formación profesional del psicólogo educativo en México. Sinéctica, 25, 3-13.

Heath, C., Hindmarsh, J. y Luff, P. (2010). Video in qualitative research: Analysing social interaction in everyday life. Londres: Sage.

Heritage, J. y Raymond, G. (2005). The Terms of Agreement: Indexing Epistemic Authority and Subordination in Assessment Sequences. Social Psychology Quarterly, 68, 15-38.

Hernández, G. (2007). Una reflexión crítica sobre el devenir de la psicología de la educación en México. Perfiles Educativos, XXIX(117), 7-40.

Lerner, G., Bolden, G., Hepburn, A. y Mandelbaum, J. (2012). Reference recalibration repairs: Adjusting the precision of formulations for the task at hand. Research on Language \& Social Interaction, 45(2), 191-212.

Macbeth, D. (2003). Hugh Mehan's learning lessons reconsidered: On the differences between the naturalistic and critical analysis of classroom discourse. American Educational Research Journal, 40(1), 239-280.

Martínez, F. y Mercado, A. (2015). Estudios sobre prácticas de evaluación en el aula: Revisión de la literatura. Revista Electrónica de Investigación Educativa, 17(1), 17-32.

Potter, J. (2012). Discourse analysis and discursive psychology. En H. Cooper (Ed.), APA Handbook of research methods in psychology: Vol. 2. Quantitative, qualitative, neuropsychological, and biological (pp. 111-130). Washington, DC: American Psychological Association Press.

Ravela, P., Picaroni, B. y Loureiro, G. (2017). ¿Cómo mejorar la evaluación en el aula?: Reflexiones y propuestas de trabajo para docentes. Ciudad de México: Instituto Nacional para la Evaluación de la Educación.

Raymond, G. y Heritage, J. (2006). The epistemics of social relations: Owning grandchildren. Language in Society, 35, 677-705.

Rodríguez, F. y Seda, I. (2013). El papel de la participación de estudiantes de Psicología en escenarios de práctica en el desarrollo de su identidad profesional. Perfiles Educativos, 35, 82-99.

Schegloff, E. (1996). Some practices for referring to persons in talk-in-interaction: A partial sketch of a systematics. En B. Fox (Ed.), Studies in anaphora (pp. 437-485). Amsterdam: John Benjamins Publishing Co. 
Stivers, T., Mondada, L. y Steensig, J. (2011). Knowledge, morality and affiliation in social interaction. En T. Stivers, L. Mondada y J. Steensig (Eds.), The morality of knowledge in conversation (pp. 3-24). Cambridge: Cambridge University Press.

Zorrilla, M. (2017). Prólogo: Innovar desde la práctica para mejorar la evaluación en el aula. En P. Ravela, B. Picaroni y G. Loureiro, ¿Cómo mejorar la evaluación en el aula?: Reflexiones y propuestas de trabajo para docentes (pp. 11-20). Ciudad de México: Instituto Nacional para la Evaluación de la Educación.

\section{Cv de los autores}

\section{Jorge Alberto Moreno Ruiz}

Licenciado en Psicología por la Facultad de Estudios Superiores Zaragoza de la Universidad Nacional Autónoma de México (UNAM) en 2010; obtuvo el grado de M. en C. en la Especialidad en Investigaciones Educativas en el año 2013 y el grado de Dr. en C. en la misma especialidad en el año 2018, por el Departamento de Investigaciones Educativas del Centro de Investigación y de Estudios Avanzados (CINVESTAV) del Instituto Politécnico Nacional. El tema de investigación en los estudios tanto de maestría como de doctorado ha sido y es la formación de psicólogos en escenarios de trabajo ordinarios como el aula. Particularmente, ha estudiado la manera en que se enseñaaprende el enfoque de la terapia centrada en soluciones, y más generalmente, la manera en que se lleva a cabo la formación de psicólogos en la práctica. Email: psiquea24@yahoo.com.mx

\section{Antonia Candela Martín}

Con grado y estudios de posgrado en Física en la UNAM, realizó maestría y doctorado en Ciencias en la especialidad de Investigaciones Educativas en el CINVESTAV. Desde finales de los 70 ha trabajado simultáneamente en el desarrollo curricular de proyectos nacionales para la enseñanza de ciencias naturales y en investigación educativa básica. Su línea de investigación en la enseñanza de la ciencia en el aula articula un enfoque etnográfico y sociocultural con análisis del discurso desde una perspectiva etnometodológica y de sociología del conocimiento científico. Como producto del trabajo de investigación ha publicado numerosos capítulos y artículos en revistas arbitradas de prestigio internacional y cuatro libros, uno de ellos: La ciencia en el aula: los alumnos entre la argumentación y el consenso en Paidós. ORCID ID: 0000-0002-9001-4451. Email: acandela@cinvestav.mx

\section{Patricia Bañuelos Lagunes}

Licenciatura en Psicologia en la UNAM. Formación en investigación educativa. Estudios de filosofía de la ciencia. Docente de la carrera de Psicologia en la FES Zaragoza, UNAM, por 40 años. Actualmente, Profesora de Tiempo Completo Asociado C. Participación en ponencias y artículos sobre la práctica supervisada en la formación de estudiantes de educación superior. Participación en la elaboración de aplicaciones didácticas para la formación de estudiantes de educación universitaria.Email: pbl@unam.mx 